\title{
The role of pro-inflammatory cytokines and immune cells in colorectal carcinoma progression
}

\author{
HUANRAN LIU $^{1 *}$, ZHEN ZHANG $^{1 *}$, TAKAFUMI TABUCHI ${ }^{2}$, SHOUYU WANG $^{1}$ and JIANGNING WANG ${ }^{3}$ \\ ${ }^{1}$ Department of Surgery, First Affiliated Hospital of Dalian Medical University, Dalian 116011, P.R. China; \\ ${ }^{2}$ Department of Surgery, Kasumigaura Hospital, Tokyo Medical University, Ibaragi 300-0395, Japan; \\ ${ }^{3}$ Department of Reparative and Reconstructive Surgery, Luhe Hospital, \\ Capital Medical University, Beijing 101100, P.R. China
}

Received September 21, 2012; Accepted January 7, 2013

DOI: $10.3892 / \mathrm{ol} .2013 .1176$

\begin{abstract}
Cytokines exhibit a pleiotropic effect in the regulation of the immune cell function, tumor growth and antitumor immune responses. A total of 30 patients with colorectal carcinoma were enrolled on this study and their levels of interleukin (IL)-1 $\beta$, IL-6, IL-8, tumor necrosis factor (TNF)- $\alpha$, serum granulocyte colony-stimulating factor (sG-CSF) and serum macrophage colony-stimulating factor (sM-CSF) were measured preoperatively using ELISA. Tumor-infiltrating granulocyte (TIG), tumor-associated macrophage (TAM), G-CSF and M-CSF expression in tumor cells were examined using immunostaining. This study revealed abnormal levels of cytokines in patients, including IL-1 $\beta(1 / 30,3.3 \%)$, IL-6 (16/30 53.3\%), IL-81 (15/30, 50\%), TNF- $\alpha$ (4/21, 19\%), sG-CSF $(17 / 30,56.7 \%)$ and sM-CSF (4/21, 19\%). There was a positive linear correlation between IL-6 and sM-CSF $(\mathrm{P}=0.017, \mathrm{R}=0.517)$. $\mathrm{sG}-\mathrm{CSF}$ was significantly associated with a deeper tumor invasion $(\mathrm{P}=0.039)$ and a more advanced tumor stage $(\mathrm{P}=0.023)$. The granulocyte/lymphocyte $(\mathrm{G} / \mathrm{L})$ ratio was associated with abnormal levels of sG-CSF. Logistic univariate analysis revealed that TIGs were a risk factor for lymph node metastasis (0.019) and TAMs were a risk factor for depth of invasion (0.029), but this was not confirmed in logistic
\end{abstract}

Correspondence to: Dr Shouyu Wang, Department of Surgery, First Affiliated Hospital of Dalian Medical University, Zhongshan Road 222, Xigang, Dalian 116011, P.R. China

E-mail: wangshouyu666@126.com

Dr Jiangning Wang, Department of Reparative and Reconstructive Surgery, Luhe Hospital, Capital Medical University, Xinhuanan Street 54, Tongzhou, Beijing 101100, P.R. China

E-mail: dldyzz@126.com

*Contributed equally

Keywords: cytokine, colorectal carcinoma, granulocyte/lymphocyte ratio, interleukin-6, granulocyte colony-stimulating factor, macrophage-colony stimulating factor multivariate analysis. In conclusion, IL-6, IL-8, sM-CSF and sG-CSF may indirectly promote tumor growth, progression and metastasis by changing the leukocyte populations in the blood and the tumor microenvironment.

\section{Introduction}

Cytokines exhibit pleiotropic effects and are crucial for the regulation of cell growth and differentiation. Previous studies have evaluated the cytokine network, which is involved in the local inflammatory and immune responses against tumors (1-4). Cytokines from tumors may either regulate tumor growth or modify the antitumor immune responses (5-8). Tumor and immune cells are capable of producing cytokines, however the key difference between these two cell types is that cytokines produced by tumor cells are not regulated.

Tumor cells are known to produce various cytokines and chemokines that attract leukocytes and promote their transformation into one of the diverse leukocyte populations, mainly granulocytes, monocytes/macrophages, dendritic cells (DCs) and lymphocytes. Subsequently, each population is able to produce an array of cytokines to allow it to escape the immune control of the host $(9,10)$. Cytokines and chemokines from tumor cells also genetically alter epithelial cells and a variety of 'normal' cells, including endothelial cells, which form the tumor vasculature, fibroblasts and inflammatory cells (e.g., lymphocytes, macrophages, mast cells and granulocytes), thus building a supportive microenvironment $(11,12)$.

In the present study, we measured the level of pro-inflammatory cytokines, tumor infiltrating granulocytes (TIGs) and tumor-associated macrophages (TAMs), determined the granulocyte/lymphocyte $(\mathrm{G} / \mathrm{L})$ ratio and examined the expression of granulocyte colony-stimulating factor (tG-CSF) and macrophage colony-stimulating factor (tM-CSF) in tumor cells to evaluate their role in tumor progression.

\section{Patients and methods}

Patient and tumor specimens. A total of 30 patients with primary colorectal carcinoma, who underwent curative surgical treatment between January 2008 and December 2011 


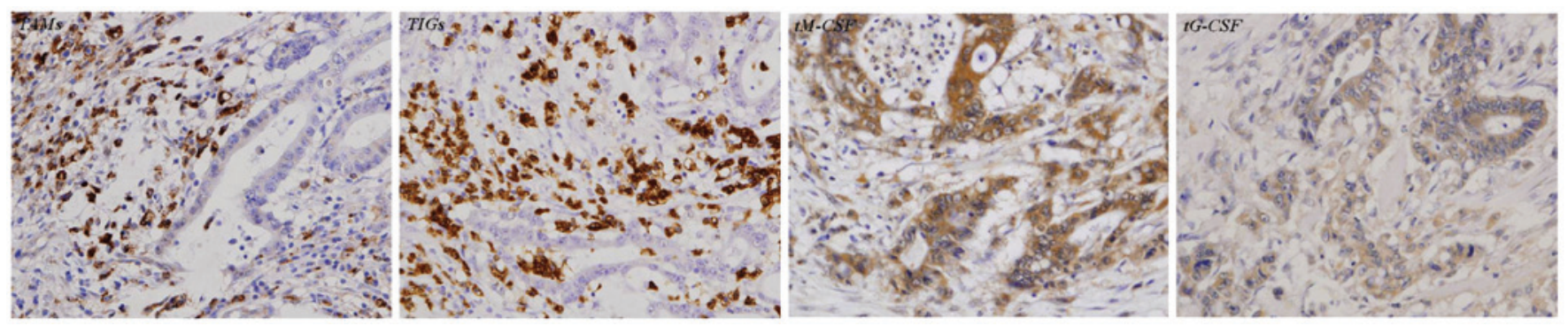

Figure 1. Immunohistochemical assay for TAMs, TIGs, tM-CSF and tG-CSF. TAMs, tumor-associated macrophages; TIGs, tumor-infiltrating granulocytes; tM-CSF, macrophage colony-stimulating factor expression in tumor cells; tG-CSF, granulocyte colony-stimulating factor expression in tumor cells.

at the Department of Surgery, First Affiliated Hospital of Dalian Medical University, Dalian, China, were included in the study. All patients received fasting hemospasia to determine the quantity of cytokines preoperatively. The tumors were histopathologically classified according to the 1997 tumor node metastasis (TNM) classification, as recommended by the International Union Against Cancer (5th edition). None of the patients had received chemotherapy or radiotherapy prior to surgery.

ELISA. The levels of immunoreactive serum G-CSF (sG-CSF), sM-CSF, interleukin (IL)-1 $\beta$, IL-6, IL-8 and tumor necrosis factor (TNF)- $\alpha$ were measured using ELISA. The following commercially available ELISA kits were used: IL-1 $\beta$, IL-6, TNF- $\alpha$, M-CSF and G-CSF (R\&D systems, Minneapolis, MN, USA) and IL-8 (Biosource Europe S.A., Nivelles, Belgium). The procedures for the cytokine assays were carried out according to the manufacturer's instructions.

\section{Immunohistochemical analysis of tumor tissues}

Detection of TIGs and TAMs. Immunostaining procedures for TIGs and TAMs were performed using the EnVision ${ }^{\mathrm{TM}}+/ \mathrm{HRP}$ method (Dako, Carpinteria, CA, USA) with heat-induced antigen retrieval. Paraffin sections $(4 \mu \mathrm{m})$ containing the tumor margin were applied. TIGs and TAMs were detected with mouse anti-granulocyte (clone SPM250, 1:50; Spring Bioscience, Fremonet, Germany) and monoclonal mouse anti-CD68 (clone KP1, 1:200; Dako) antibodies. Negative control sections were stained by omitting the primary antibody.

Detection of M-CSF expression ( $t M-C S F$ ) and G-CSF expression $(t G-C S F)$ in tumor cells. tM-CSF and tG-CSF in colorectal carcinomas were detected by immunohistochemical staining with monoclonal anti-G-CSF (clone 4-12-2, 1:100; Immuno-Biological Laboratories, Gunma, Japan) and monoclonal anti-M-CSF (clone EP1179Y, 1:100; Epitomics, Burlingame, CA, USA) antibodies. Negative control sections were stained by omitting the primary antibody. Specimens were considered to be positive for tG-CSF and tM-CSF when $\geq 20 \%$ of the tumor cells exhibited positive immunoreactivity.

Statistical analysis. All data, including stage of disease and pathological factors, were obtained from clinical and pathological records. Pearson's correlation coefficient and Spearman's correlation were used to assess the correlation between related variables. Significant differences were determined using the Kruskal-Wallis test. Binary logistic

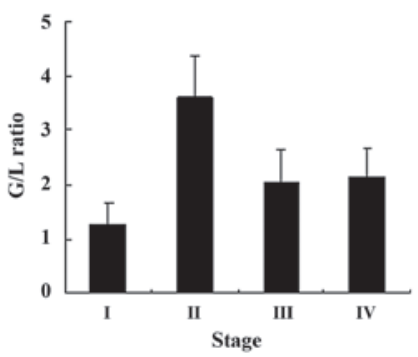

Figure 2. Correlation between the tumor stage and G/L ratio. G/L, granulocyte/lymphocyte.

regression analysis was employed to determine the significant predictors for lymph node metastasis and depth of tumor invasion. $\mathrm{P}<0.05$ was considered to indicate a statistically significant difference.

\section{Results}

Cytokine production in colorectal patients. A total of 30 patients underwent preoperative blood sampling, however the data for SM-CSF and TNF- $\alpha$ were lost for 9 patients. Abnormal levels (a higher than normal value) of IL-1 $\beta$, IL-6, IL- 8 , TNF- $\alpha$, sG-CSF and sM-CSF were detected in $1 / 30$ (3.3\%), 16/30 (53.3\%), 15/30 (50\%), 4/21(19\%), 17/30 (56.7\%) and 4/21 (19\%) cases, respectively.

Detection of TIGs and TAMs and their correlation. The average number of TIGs and TAMs was $47.3 \pm 26.1$ and $32.5 \pm 33.8$ per $10 \times 40 \mathrm{hpf}$, respectively. TIGs were mainly located at the site of tumor invasion, however TAMs were located in areas of tumor invasion and the stroma (Fig. 1). Granulocytes and macrophages in necrotic regions were excluded from the statistical analysis. No significant positive correlation was identified between TIGs and TAMs $(\mathrm{P}=0.58$, Table I).

Correlation between cytokines. We analyzed the correlation between all the cytokines which feature in this study. Pearson's correlation coefficient test revealed that there was a positive linear correlation between IL- 6 and sM-CSF $(\mathrm{P}=0.017$, $\mathrm{R}=0.517$ ) and Spearman's correlation test revealed that there was a significant correlation between IL-6 and sG-CSF ( $\mathrm{P}=0.045, \mathrm{R}=0.369$ ). However, no significant correlation was identified between the remaining combinations of cytokines (Table I). 
Table I. Correlation between cytokines and G/L ratio.

\begin{tabular}{|c|c|c|c|c|c|c|c|c|}
\hline Variable & IL-6 & sM-CSF & sG-CSF & IL-8 & IL-1 $\beta$ & TNF- $\alpha$ & $\mathrm{G} / \mathrm{L}$ ratio & TIGs \\
\hline \multicolumn{9}{|l|}{ sM-CSF } \\
\hline $\mathrm{R}$ & $0.517^{\mathrm{b}}$ & & & & & & & \\
\hline P-value & 0.017 & & & & & & & \\
\hline \multicolumn{9}{|l|}{ sG-CSF } \\
\hline $\mathrm{R}$ & $0.369^{\mathrm{a}}$ & & & & & & & \\
\hline P-value & 0.045 & 0.56 & & & & & & \\
\hline \multicolumn{9}{|l|}{ IL-8 } \\
\hline \multicolumn{9}{|l|}{$\mathrm{R}$} \\
\hline P-value & 0.63 & 0.75 & 0.37 & & & & & \\
\hline \multicolumn{9}{|l|}{ IL-1 $\beta$} \\
\hline \multicolumn{9}{|l|}{$\mathrm{R}$} \\
\hline P-value & 0.90 & 0.99 & 0.56 & 0.43 & & & & \\
\hline \multicolumn{9}{|l|}{ TNF- $\alpha$} \\
\hline \multicolumn{9}{|l|}{$\mathrm{R}$} \\
\hline P-value & 0.44 & 0.69 & 0.44 & 0.89 & 0.62 & & & \\
\hline \multicolumn{9}{|l|}{$\mathrm{G} / \mathrm{L}$ ratio } \\
\hline \multicolumn{9}{|l|}{$\mathrm{R}$} \\
\hline P-value & 0.24 & 0.89 & 0.45 & 0.58 & 0.65 & 0.67 & & \\
\hline \multicolumn{9}{|l|}{ TIGs } \\
\hline $\mathrm{R}$ & & & & & & $0.43^{\mathrm{a}}$ & & \\
\hline P-value & 0.945 & 0.320 & 0.364 & 0.445 & 0.297 & 0.050 & 0.888 & \\
\hline \multicolumn{9}{|l|}{ TAMs } \\
\hline $\mathrm{R}$ & & & & $0.32^{\mathrm{a}}$ & & & & \\
\hline P-value & 0.64 & 0.75 & 0.33 & 0.09 & 0.67 & 0.49 & 0.40 & 0.579 \\
\hline
\end{tabular}

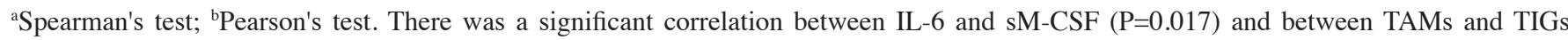
$(\mathrm{P}=0.024)$. IL, interleukin; sM-CSF, serum macrophage colony-stimulating factor; sG-CSF, serum granulocyte colony-stimulating factor; TNF- $\alpha$, tumor necrosis factor- $\alpha$; G/L ratio, granulocyte/lymphocyte ratio; TIGs, tumor-infiltrating granulocytes; TAMs, tumor-associated macrophages.

Association of $G / L$ ratio with tumor stage and cytokines. We investigated the association between the tumor stage/ cytokines and the $\mathrm{G} / \mathrm{L}$ ratio; a significant correlation was identified for tumor stage ( $\mathrm{P}=0.037$, Fig. 2), however, the $\mathrm{G} / \mathrm{L}$ ratio was not associated with the levels of cytokines (Table I).

Association of cytokines with TIGs and TAMs. The patients were divided into groups for normal or abnormal cytokine levels. Kruskal-Wallis testing revealed that the level of sG-CSF was significantly increased in the abnormal IL-6 level group ( $\mathrm{P}=0.022)$, the $\mathrm{G} / \mathrm{L}$ ratio was significantly increased in the abnormal sG-CSF level group $(\mathrm{P}=0.049)$, the level of IL-8 was significantly decreased in the abnormal sG-CSF level group $(\mathrm{P}=0.026)$ and TAM levels were significantly increased in the abnormal IL-8 level group $(\mathrm{P}=0.037$; Fig. 3).

Association of cytokines, TIGs and TAMs with clinicopathological factors. Analysis revealed that sG-CSF was significantly associated with the depth of tumor invasion $(\mathrm{P}=0.039)$ and a more advanced tumor stage $(\mathrm{P}=0.023)$. However, significant correlation was not identified between cytokines and lymph node metastasis and between TIGs or
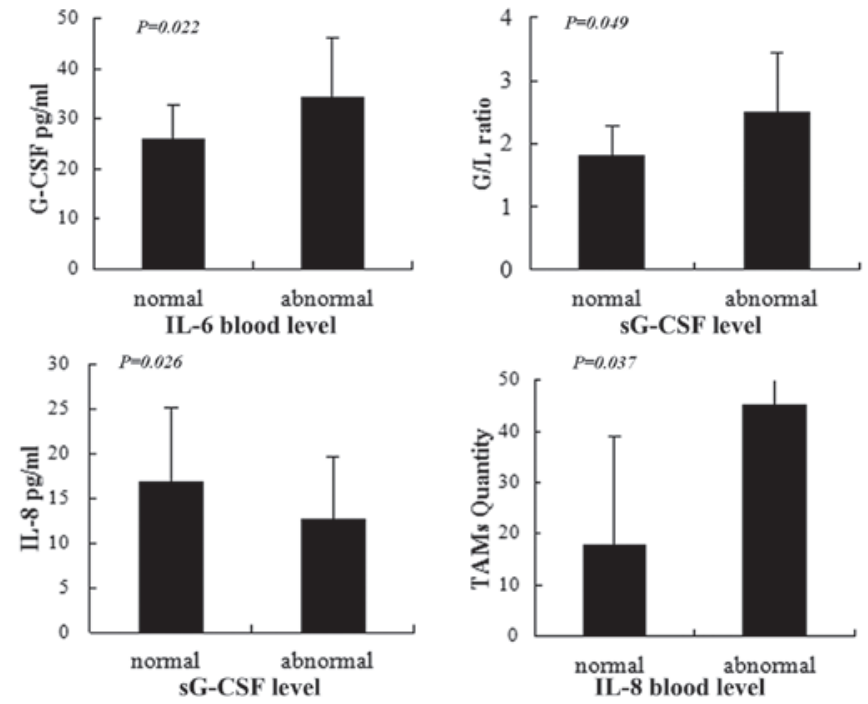

Figure 3. Effects of cytokine levels. Kruskal-Wallis testing revealed that sG-CSF was significantly increased in the abnormal IL-6 level group $(\mathrm{P}=0.022)$; the $\mathrm{G} / \mathrm{L}$ ratio was associated with abnormal levels of sG-CSF $(\mathrm{P}=0.049)$; IL-8 was significantly decreased in the high sG-CSF level group $(\mathrm{P}=0.026)$; TAMs were associated with abnormal levels of IL-8 $(\mathrm{P}=0.037)$. sG-CSF, serum granulocyte colony-stimulating factor; $\mathrm{G} / \mathrm{L}$, granulocyte/ lymphocyte; IL, interleukin, TAMs, tumor-associated macrophages. 
Table II. Association of cytokines with clinicopathological factors.

Cytokines according to tumor features $(\mathrm{pg} / \mathrm{ml})$

\begin{tabular}{|c|c|c|c|c|c|c|c|c|c|}
\hline \multirow[b]{2}{*}{ Variable } & \multicolumn{3}{|c|}{ Lymph node metastasis } & \multicolumn{3}{|c|}{ Depth of invasion } & \multicolumn{3}{|c|}{ Tumor stage } \\
\hline & Absent & Present & P-value & $\begin{array}{c}\text { Under } \\
\text { muscle layer }^{\mathrm{a}}\end{array}$ & $\begin{array}{c}\text { Beyond } \\
\text { muscle layer }\end{array}$ & P-value & $\begin{array}{l}\text { Stage } \\
0 \text { and I }\end{array}$ & $\begin{array}{l}\text { Stage II, } \\
\text { III and IV }\end{array}$ & P-value \\
\hline sG-CSF & $26.5 \pm 7.6$ & $32.2 \pm 11.2$ & NS & $23.9 \pm 4.1$ & $31.4 \pm 11.1$ & 0.039 & $22.7 \pm 4.1$ & $32.4 \pm 10.7$ & 0.023 \\
\hline sM-CSF & $504 \pm 241.4$ & $359.2 \pm 172.5$ & NS & $407.3 \pm 208.82$ & $399 \pm 138.9$ & NS & $470.7 \pm 295.1$ & $389.0 \pm 188.9$ & NS \\
\hline IL-1 $\beta$ & $0.32 \pm 0.2$ & $0.28 \pm 0.2$ & NS & $0.4 \pm 0.3$ & $0.2 \pm 0.1$ & 0.086 & $0.35 \pm 0.2$ & $0.28 \pm 0.2$ & NS \\
\hline IL-6 & $23.3 \pm 62.5$ & $5.0 \pm 7.1$ & NS & $2.4 \pm 1.4$ & $12.9 \pm 17.2$ & NS & $2.52 \pm 2.1$ & $12.48 \pm 38.4$ & NS \\
\hline IL-8 & $10.9 \pm 4.2$ & $16.1 \pm 14.4$ & NS & $9.9 \pm 2.0$ & $16.0 \pm 8.9$ & NS & $10.2 \pm 2.5$ & $15.6 \pm 13.6$ & NS \\
\hline TNF- $\alpha$ & $1.0 \pm 0.6$ & $1.3 \pm 0.4$ & NS & $1.0 \pm 0.3$ & $1.2 \pm 0.6$ & NS & $1.0 \pm 0.5$ & $1.2 \pm 0.8$ & NS \\
\hline TIGs & $33.9 \pm 12.5$ & $53.1 \pm 22.9$ & 0.065 & $40.3 \pm 16.5$ & $49.5 \pm 23.1$ & NS & $40.6 \pm 18.2$ & $50.2 \pm 21.9$ & NS \\
\hline TAMs & $32.4 \pm 16.8$ & $32.5 \pm 28.3$ & NS & $42.6 \pm 22.5$ & $29.4 \pm 25.5$ & NS & $46.2 \pm 39.0$ & $26.6 \pm 20.4$ & NS \\
\hline
\end{tabular}

Results are presented as mean $\pm \mathrm{SD}$. Includes muscle layer. sG-CSF was significantly associated with deeper tumor invasion $(\mathrm{P}=0.039)$ and more advanced tumor stage $(\mathrm{P}=0.023, \mathrm{Rs}=0.375) . \mathrm{GG}-\mathrm{CSF}$, serum granulocyte colony-stimulating factor; sM-CSF, serum macrophage colony-stimulating factor; IL, interleukin; TNF- $\alpha$, tumor necrosis factor- $\alpha$; TIGs, tumor-infiltrating granulocytes; TAMs, tumor-associated macrophages; NS, not significant.
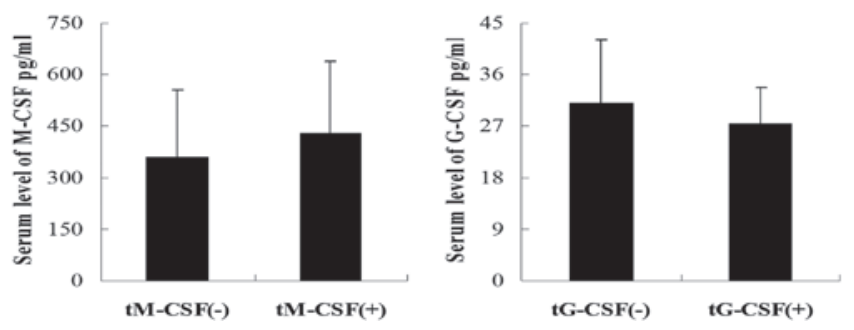

Figure 4. Correlation between CSF expression in tumor cells and the level of serum CSF. There was no significant correlation between tM-CSF and the level of sM-CSF $(\mathrm{P}=0.442)$ and between $\mathrm{tG}-\mathrm{CSF}$ and the level of sG-CSF $(\mathrm{P}=0.498)$. CSF, colony-stimulating factor; tM-CSF, macrophage CSF expression in tumor cells; sM-CSF, serum macrophage CSF; tG-CSF, granulocyte CSF expression in tumor cells; sG-CSF, serum granulocyte CSF.

TAMs and tumor stage, depth of invasion and lymph node metastasis (Table II).

Detection of $t M-C S F$ and $t G-C S F$ by immunostaining. Immunostaining, which used antibodies against tM-CSF and tG-CSF (Fig. 1), revealed that tM-CSF and tG-CSF in tumor cells was detected in $17 / 30(56.7 \%)$ and $5 / 30(16.7 \%)$ of cases, respectively. No significant correlation was identified between CSF expression in tumor cells and the serum levels of CSF for $\mathrm{M}-\mathrm{CSF}(\mathrm{P}=0.442)$ or $\mathrm{G}-\mathrm{CSF}(\mathrm{P}=0.498$; Fig. 4).

Logistic regression analysis of risk factors for lymph node metastasis and depth of tumor invasion. Logistic univariate analysis revealed that TIGs were correlated with lymph node metastasis, however, in logistic multivariate analysis, they were not identified as a significant independent risk factor for lymph node metastasis $(\mathrm{P}=0.069)$. Logistic univariate analysis also revealed that levels of TAMs were correlated with depth of invasion, however, in logistic multivariate analysis, they were not identified as an independent risk factor for depth of invasion $(\mathrm{P}=0.063$; Table III).
Table III. Logistic regression analysis of risk factors for lymph node metastasis and depth of invasion.

\begin{tabular}{|c|c|c|c|}
\hline \multirow[b]{2}{*}{ Variable } & \multirow{2}{*}{$\begin{array}{l}\text { Univariate } \\
\text { P-value }\end{array}$} & \multicolumn{2}{|c|}{ Multivariate } \\
\hline & & P-value & $\mathrm{RR}(95 \% \mathrm{CI})$ \\
\hline \multicolumn{4}{|c|}{ Lymph node metastasis ${ }^{\mathrm{a}}$} \\
\hline G-CSF & 0.269 & & \\
\hline M-CSF & 0.124 & & \\
\hline IL-1 $\beta$ & 0.731 & & \\
\hline IL-6 & 0.15 & & \\
\hline IL-8 & 0.703 & & \\
\hline TNF- $\alpha$ & 0.452 & & \\
\hline TIGs & 0.019 & 0.069 & $1.087(0.994-1.188)$ \\
\hline TAMs & 0.353 & & \\
\hline sG-CSF & 0.517 & & \\
\hline sM-CSF & 0.676 & & \\
\hline \multicolumn{4}{|c|}{ Depth of invasion ${ }^{\mathrm{b}}$} \\
\hline G-CSF & 0.083 & 0.071 & $1.301(0.978-1.733)$ \\
\hline M-CSF & 0.939 & & \\
\hline $\mathrm{IL}-1 \beta$ & 0.083 & & \\
\hline IL-6 & 0.517 & & \\
\hline IL-8 & 0.567 & & \\
\hline $\mathrm{TNF}-\alpha$ & 0.59 & & \\
\hline TIGs & 0.262 & & \\
\hline TAMs & 0.029 & 0.063 & $0.64(0.927-1.002)$ \\
\hline sG-CSF & 0.619 & & \\
\hline sM-CSF & 0.422 & & \\
\hline
\end{tabular}

a -2 Log likelihood $=17.811$, Nagel kerke $R^{2}=0.422$; ${ }^{\text {b }} 2$ Log likelihood $=9.362$, Nagel kerke $\mathrm{R}^{2}=0.659$. CI, confidence interval; G-CSF, granulocyte colony-stimulating factor; M-CSF, macrophage colony-stimulating factor; IL, interleukin; TNF- $\alpha$, tumor necrosis factor- $\alpha$; TIGs, tumor-infiltrating granulocytes; TAMs, tumor-associated macrophages; sG-CSF, serum G-CSF; sM-CSF, serum M-CSF. 


\section{Discussion}

This study aimed to clarify the correlation between proinflammatory cytokines, immune cells and tumors. This has previously been investigated by numerous studies, which showed that cytokines regulate cell growth and, more importantly, cell proliferation, and they may be produced by tumor or immune cells. However, the role of cytokines in inducing granulocytes or macrophages to become involved in the antitumor immune response was unclear.

G-CSF is produced by normal monocytes, macrophages and granulocytes. In the present study, we identified abnormal levels of sG-CSF in 17/30 (56.7\%) cases, however, only a few cases of G-CSF-producing colorectal carcinomas have ever been reported. We also examined tG-CSF expression in tumor cells using immunohistochemical staining. A reaction for tG-CSF was observed in only 5 cases and this was not associated with the level of $\mathrm{sG}-\mathrm{CSF}(\mathrm{P}=0.498)$. The levels of immunoreactive sM-CSF, IL-1 $\beta$, IL- 6, IL- 8 and TNF- $\alpha$ were measured by ELISA and a positive linear correlation was identified between the levels of IL-6 and sM-CSF. The level of sG-CSF was significantly increased in the abnormal IL-6 level group. M-CSF expression in tumor cells was examined using immunostaining and immunoreactivity was observed in $17 / 30(56.7 \%)$ cases, which is similar to results from previous studies on M-CSF production by tumor cells (13-16). However, only 4 patients (19\%) had abnormal levels of sM-CSF and there was no significant correlation between tM-CSF and sM-CSF ( $\mathrm{P}=0.442)$. A study by Ashizawa et al (17) showed that immunohistochemical staining revealed positive results for IL-6 expression in the cytoplasm of colorectal cancer cells in patients with a high serum level of IL-6, however there was no evidence of positive results for IL-6 expression in patients with a normal serum level of IL-6. This suggests that IL-6 may be able to induce the production of CSFs from numerous types of cells, in addition to tumors (16). IL-6 is a pleiotropic cytokine which is secreted by a wide variety of cell types, including lymphocytes, monocytes and tumor cells $(17,18)$. Sato et al (19) demonstrated that IL-6, sG-CSF and sM-CSF appear to contribute to neutrophilia in cases of anaplastic thyroid carcinoma. A high count of peripheral neutrophils was correlated with poor prognosis in patients with a variety of cancer types, including breast, head and neck cancer and sarcoma (20-24).

Therefore, we measured the G/L ratio, which represents the relative number of these two major leukocyte populations, indicates fluctuations in their numbers and reveals their potential impact on the progression and prognosis of cancer. Hence, the $\mathrm{G} / \mathrm{L}$ ratio, which is easily measured in a clinical setting, is a valuable indicator of tumor progression (25) and is useful for selecting patients who are appropriate for surgery (26). Our results revealed that the $\mathrm{G} / \mathrm{L}$ ratio was significantly associated with a more advanced tumor stage $(\mathrm{P}=0.037)$ and it was significantly increased in the abnormal sG-CSF level group. Thus it is hypothesized that sG-CSF, sM-CSF and IL-6 may indirectly assist tumor growth and progression by neutrophilia. Next, we analyzed the correlation between cytokines and tumor features. The results revealed that sG-CSF levels were positively associated with deeper tumor invasion and a more advanced tumor stage.
In carcinogenesis, cytokines and chemokines from tumor cells are able to build a supportive microenvironment and induce inflammatory cells, which contribute to tumor growth, progression and metastasis (27-29). In this study, measuring the quantity of TIGs and TAMs revealed that levels of TIGs were increased with tumor progression, however TAM levels were decreased. Next, the correlation between cytokines and TIGs or TAMs was tested; a significantly positive correlation was identified between IL- 8 and TAMs and the IL- 8 level was significantly decreased in the high sG-CSF level group. Based on the above-mentioned results for sG-CSF, we hypothesized that IL-8 should decrease with tumor progression, however, the present study failed to demonstrate this. This may be due to the complicated role of TAMs in tumor progression; since TAMs have a dual role in neoplasms, it is difficult to accurately evaluate the correlation between IL-8 and TAMs. IL-8 is known to recruit inflammatory neutrophils and promote the interaction between tumor cells and inflammatory cells (30) and it is also a potent angiogenic and growth factor in malignant tumors $(31,32)$. Therefore, we hypothesized that IL- 8 may selectively induce the infiltration of immune cells into tumors, in order to assist tumor progression. The present study evaluated the risk factors for lymph node metastasis and depth of invasion by logistic regression analysis. Univariate analysis revealed that TIGs were correlated with lymph node metastasis, however, logistic multivariate analysis determined that $\mathrm{P}=0.069$, which is close to 0.05 . Further univariate analysis revealed that TAMs were correlated with depth of invasion and multivariate analysis revealed that $\mathrm{P}=0.063$, which is also close to 0.05 . It is possible that significant results were not obtained due to the small sample size, however, we believe that TIGs and TAMs should be risk factors for lymph node metastasis and depth of invasion, respectively.

Due to the pleiotropy of cytokines, it is difficult to confirm the roles of cytokines in the growth and progression of tumors. However, the present study reveals a correlation between tumors and immune cells, which secrete cytokines, and this suggests that cytokines may indirectly promote the growth, progression and metastasis of tumors.

\section{Acknowledgements}

The authors thank Tsutomu Kohda for technical assistance.

\section{References}

1. Schoenfeld J,Jinushi M, Nakazaki Y, et al: Active immunotherapy induces antibody responses that target tumor angiogenesis. Cancer Res 70: 10150-10160, 2010.

2. Noori S, Taghikhani M, Hassan ZM, Allameha A and Mostafaei A: Tehranolide molecule modulates the immune response, reduce regulatory $\mathrm{T}$ cell and inhibits tumor growth in vivo. Mol Immunol 47: 1579-1584, 2010.

3. Lesterhuis WJ, Punt CJ, Hato SV, et al: Platinum-based drugs disrupt STAT6-mediated suppression of immune responses against cancer in humans and mice. J Clin Invest 121: 3100-3108, 2011.

4. Wieder T, Braumüller H, Kneilling M, Pichler B and Röcken M: T cell-mediated help against tumors. Cell Cycle 7: 2974-2977, 2008.

5. Forte G, Sorrentino R, Montinaro A, et al: Inhibition of CD73 improves B cell-mediated anti-tumor immunity in a mouse model of melanoma. J Immunol 189: 2226-2233, 2012. 
6. Kandasamy M, Bay BH, Lee YK and Mahendran R: Lactobacilli secreting a tumor antigen and IL15 activates neutrophils and dendritic cells and generates cytotoxic $\mathrm{T}$ lymphocytes against cancer cells. Cell Immunol 271: 89-96, 2011

7. Schwandt A, Garcia JA, Elson P, et al: Clinical and immunomodulatory effects of celecoxib plus interferon-alpha in metastatic renal cell carcinoma patients with COX-2 tumor immunostaining. J Clin Immunol 31: 690-698, 2011.

8. Yamamoto M, Kamigaki T, Yamashita K, et al: Enhancement of anti-tumor immunity by high levels of Th1 and Th17 with a combination of dendritic cell fusion hybrids and regulatory $\mathrm{T}$ cell depletion in pancreatic cancer. Oncol Rep 22: 337-343, 2009.

9. Kuper H, Adami HO and Trichopoulos D: Infections as a major preventable cause of human cancer. J Intern Med 248: 171-183, 2000.

10. Mareel M and Madani I: Tumour-associated host cells participating at invasion and metastasis: targets for therapy? Acta Chir Belg 106: 635-640, 2006.

11. de Visser KE and Coussens LM: The inflammatory tumor microenvironment and its impact on cancer development. Contrib Microbiol 13: 118-137, 2006.

12. Kenny PA and Bissell MJ: Tumour reversion: correction of malignant behavior by microenvironmental cues. Int J Cancer 107: 688-695, 2003.

13. Revoltella RP, Menicagli M and Campani D: Granulocytemacrophage colony-stimulating factor as an autocrine survival-growth factor in human gliomas. Cytokine 57: 347-359, 2012.

14. Curran CS, Evans MD and Bertics PJ: GM-CSF production by glioblastoma cells has a functional role in eosinophil survival, activation, and growth factor production for enhanced tumor cell proliferation. J Immunol 187: 1254-1263, 2011.

15. Liao HH, Wang YC, Chen MC, et al: Down-regulation of granulocyte-macrophage colony-stimulating factor by $3 \mathrm{C}$-like proteinase in transfected A549 human lung carcinoma cells. BMC Immunol 12: 16, 2011.

16. Inoue $H$, Iga $M$, Nabeta $H$, et al: Non-transmissible Sendai virus encoding granulocyte macrophage colony-stimulating factor is a novel and potent vector system for producing autologous tumor vaccines. Cancer Sci 99: 2315-2326, 2008.

17. Ashizawa T, Okada R, Suzuki Y, et al: Study of interleukin-6 in the spread of colorectal cancer: the diagnostic significance of IL-6. Acta Med Okayama 60: 325-330, 2006.

18. Bao B, Ahmad A, Kong D, et al: Hypoxia induced aggressiveness of prostate cancer cells is linked with deregulated expression of VEGF, IL- 6 and miRNAs that are attenuated by CDF. PLoS One 7: e43726, 2012.

19. Sato T, Omura M, Saito J, Hirasawa A, Kakuta Y, Wakabayashi Y and Nishikawa T: Neutrophilia associated with anaplastic carcinoma of the thyroid: production of macrophage colonystimulating factor (M-CSF) and interleukin-6. Thyroid 10: $1113-1118,2000$
20. Riesco A: Five-year cancer cure: relation to total amount of peripheral lymphocytes and neutrophils. Cancer 25: 135-140, 1970.

21. Atzpodien J, Royston P, Wandert T and Reitz M; DGCIN German Cooperative Renal Carcinoma Chemo-Immunotherapy Trials Group: Metastatic renal carcinoma comprehensive prognostic system. Br J Cancer 88: 348-353, 2003.

22. Négrier S, Escudier B, Gomez F, et al: Prognostic factors of survival and rapid progression in 782 patients with metastatic renal carcinomas treated by cytokines: a report from the Groupe Français d'Immunothérapie. Ann Oncol 13: 1460-1468, 2002.

23. Donskov F and von der Maase H: Impact of immune parameters on long-term survival in metastatic renal cell carcinoma. J Clin Oncol 24: 1997-2005, 2006.

24. Schmidt H, Suciu S, Punt CJ, et al; American Joint Committee on Cancer Stage IV Melanoma; EORTC 18951: Pretreatment levels of peripheral neutrophils and leukocytes as independent predictors of overall survival in patients with American Joint Committee on Cancer Stage IV Melanoma: results of the EORTC 18951 Biochemotherapy Trial. J Clin Oncol 25: 1562-1569, 2007.

25. Liu H, Tabuchi T, Takemura A, et al: The granulocyte/lymphocyte ratio as an independent predictor of tumour growth, metastasis and progression. Mol Med Rep 1: 699-704, 2008.

26. Ietomi K: A study on the role of granulocytes in carcinoma-bearing hosts - G/L ratio as a new host indicator. Nippon Gan Chiryo Gakkai Shi 25: 662-671, 1990 (In Japanese).

27. Lee YS, Choi I, Ning Y, et al: Interleukin-8 and its receptor CXCR2 in the tumour microenvironment promote colon cancer growth, progression and metastasis. Br J Cancer 106: 1833-1841, 2012.

28. Jung MY, Kim SH, Cho D and Kim TS: Analysis of the expression profiles of cytokines and cytokine-related genes during the progression of breast cancer growth in mice. Oncol Rep 22: 1141-1147, 2009.

29. Balkwill $\mathrm{F}$ and Mantovani A: Inflammation and cancer: back to Virchow? Lancet 357: 539-545, 2001.

30. Arenberg DA, Kunkel SL, Polverini PJ, Glass M, Burdick MD and Strieter RM: Inhibition of interleukin-8 reduces tumorigenesis of human non-small cell lung cancer in SCID mice. J Clin Invest 97: 2792-2802, 1996.

31. Ferrara N and Davis-Smyth T: The biology of vascular endothelial growth factor. Endocr Rev 18: 4-25, 1997.

32. Mizukami Y, Jo WS, Duerr EM, et al: Induction of interleukin-8 preserves the angiogenic response in HIF-1alpha-deficient colon cancer cells. Nat Med 11: 992-997, 2005. 